(2) Open Access Full Text Article

\title{
Antipsychotic monotherapy among outpatients with schizophrenia treated with olanzapine or risperidone in Japan: a health care database analysis
}

\author{
This article was published in the following Dove Press journal: \\ Neuropsychiatric Disease and Treatment \\ 16 June 2012 \\ Number of times this article has been viewed
}

\author{
Wenyu $\mathrm{Ye}^{1}$ \\ Haya Ascher-Svanum² \\ Yuka Tanji ${ }^{3}$ \\ Jennifer A Flynn ${ }^{3}$ \\ Michihiro Takahashi, $i^{3,4}$ \\ Robert R Conley ${ }^{2}$ \\ 'Lilly Suzhou Pharmaceutical Co, Ltd, \\ Shanghai, People's Republic of China; \\ ${ }^{2}$ Eli Lilly and Company, Indianapolis, \\ IN, USA; ${ }^{2}$ Lilly Research Laboratories \\ Japan, Eli Lilly Japan KK, Kobe, Japan; \\ ${ }^{4}$ Terauchi-Takahashi Psychiatric Clinic, \\ Ashiya, Japan
}

Purpose: Antipsychotic monotherapy is often recommended over antipsychotic polypharmacy because of fewer adverse events, reduced treatment complexity, and lower medication cost. This study compared the rate and the duration of antipsychotic monotherapy following initiation of olanzapine or risperidone in the treatment of outpatients with schizophrenia in Japan.

Methods: Outpatients diagnosed with schizophrenia in the Japan Medical Data Center database were identified using International Statistical Classification of Diseases and Related Health Problems, 10th Revision, diagnosis codes. Patients were between 20 and 65 years old, initiated on olanzapine or risperidone therapy between August 2003 and July 2008, and continuously enrolled during the 6 months prior to and the 12 months following the initiation date. Antipsychotic polypharmacy was defined as concurrent use of two or more antipsychotics. The probability of monotherapy during the 12-month follow-up period was assessed using a propensity score-adjusted generalized estimating equation model. Duration of monotherapy was contrasted using a propensity score-adjusted bootstrapping model.

Results: After applying all inclusion and exclusion criteria, the final analytic sample consisted of 332 olanzapine- and 496 risperidone-treated outpatients. At treatment initiation, $61.5 \%$ of the olanzapine-treated patients and $45.6 \%$ of the risperidone-treated patients received antipsychotic monotherapy $(P<0.001)$. After correcting for background differences, monotherapy was more common among olanzapine-treated patients $(P=0.001)$. In addition, olanzapine was used as monotherapy for a longer duration $(P=0.006)$.

Conclusion: Consistent with prior global research, this retrospective naturalistic study of schizophrenia outpatients in Japan found that olanzapine is more likely to be used as monotherapy and to be used as monotherapy for a longer duration than risperidone.

Keywords: epidemiologic studies, polypharmacy, monotherapy duration, outpatient care, Japan

\section{Introduction}

Antipsychotic medications have been a core treatment modality for patients with schizophrenia over the past 40 years, with antipsychotic monotherapy considered the treatment of choice. ${ }^{1}$ Despite consistent recommendations for antipsychotic monotherapy, antipsychotic polypharmacy, the concomitant use of two or more antipsychotics, is commonplace in the treatment of schizophrenia. ${ }^{2-7}$ Antipsychotic polypharmacy seems to be increasing over time as additional antipsychotic treatment options become available. , $^{8}$ 
Antipsychotic polypharmacy appears to be used to improve efficacy after partial response or nonresponse to monotherapy, ${ }^{10,11}$ but these clinical benefits have not been clearly documented. ${ }^{4,7,12,13}$ However, the disadvantages are clear: antipsychotic polypharmacy has a higher risk of adverse events and potential drug-drug interactions; ${ }^{11}$ it increases complexity, making assessment of the medication regimen and management of future symptom exacerbations more difficult; ${ }^{14}$ and it increases antipsychotic treatment costs at a time of growing budget constraints. ${ }^{14-19}$

The prevalence of antipsychotic polypharmacy has been reported to range between $13 \%$ and $70 \%$, depending on the study population, country, year, methodology, and duration. ${ }^{6,7,20-26}$ A prospective, observational, noninterventional study in the United States (US) ${ }^{27}$ found that over a 12 -month period, $58 \%$ of the patients had at least one period of antipsychotic polypharmacy lasting 60 consecutive days or longer. The rate of antipsychotic polypharmacy has been found to be higher in Japan than in the US. ${ }^{28}$ A Japanese national cross-sectional survey of 9325 inpatients with schizophrenia revealed that $67 \%-70 \%$ were treated with more than one antipsychotic, and $75.6 \%$ of those treated with an atypical antipsychotic were also treated with another antipsychotic. ${ }^{25,26}$ The very high rate of antipsychotic polypharmacy has led the Japanese Ministry of Health, Labour and Welfare to reward physicians who reduce their patients' atypical antipsychotic polypharmacy.

Prior research has identified multiple factors that predict antipsychotic polypharmacy use. Antipsychotic polypharmacy has been associated with greater illness severity, longer illness duration, comorbid depression, and treatment with typical antipsychotics, as well as the use of certain atypical antipsychotics. ${ }^{4,7,10}$ The rate and duration of antipsychotic polypharmacy differs among atypical antipsychotics, ${ }^{7}$ with olanzapine-initiated patients more likely to be treated with monotherapy than those treated with other atypical antipsychotics. ${ }^{16,23,27,29-32}$ Prior research has shown a higher rate and a longer duration of antipsychotic monotherapy with olanzapine than risperidone in the treatment of patients with schizophrenia in the US ${ }^{27}$ and in Europe. ${ }^{32}$ However, it is unclear whether these prior findings can generalize across world geographies, especially to the Japanese health care system, where antipsychotic polypharmacy appears to be highly prevalent.

The objective of the current retrospective claims database analysis is to assess the rate and duration of antipsychotic polypharmacy among outpatients in Japan who were initiated on olanzapine or risperidone for the treatment of schizophrenia.
Olanzapine and risperidone are the two most frequently used atypical antipsychotics for schizophrenia in Japan. ${ }^{33}$

\section{Methods \\ Data source}

This study utilized the Japan Medical Data Centre Database (JMDC), an employment-based administrative database containing the medical and pharmacy claims from ten different payers (insurance societies). The JMDC included information on approximately 0.6 million employed individuals or their family members who were enrolled between August 2003 and July 2009.

The JMDC consisted of inpatient, outpatient, and pharmacy administrative claims. The medical claims (inpatient and outpatient) included basic demographics, diagnoses, procedures, and fees. Diagnoses were specified with International Statistical Classification of Diseases and Related Health Problems, 10th Revision (ICD-10), diagnosis codes.$^{34}$ For the medical claims, the date of service information was restricted to month and year. Pharmacy claims included the full dispensing date, the days of supply, and dosage information. Drugs were classified using the Anatomical Therapeutic Chemical Classification System codes. ${ }^{35}$

\section{Sample selection}

The initial extract of data from the JMDC database included the administrative claims between August 2003 and July 2009 for patients who had at least one diagnosis of schizophrenia (F20.0-F20.9). This initial data cut included 4861 individuals.

The primary selection criterion for this analysis was the initiation of either olanzapine or risperidone (ie, index drug) before June 30, 2008. Based on each patient's first pharmacy claim for the index drug, an index date was identified. Initiation was defined as a period of 3 months without a claim for the index drug prior to the index date. Patients were categorized into two mutually exclusive study cohorts based on the most recent initiation: risperidone or olanzapine.

The study period varied for each patient and included the 6 months preceding and the 12 months following the index date. The study period was divided into a baseline period and a treatment period, with the index date marking the first day of the treatment period.

Additional inclusion and exclusion criteria included the following: (1) patients were required to have at least one diagnosis of schizophrenia (F20.0-F20.9) during the study period; (2) patients were excluded from the study if they had ICD-10 diagnoses for any of the following conditions during 
the study period: organic mental disorders, organic brain disorders, dementia, or Alzheimer's disease; (3) patients were excluded from the analysis if they did not have at least one claim prior to and one claim after the study period (this was used as a proxy for continuous enrollment); (4) the analysis was restricted to individuals who were between 20 and 65 years of age (the lower cutoff was used to restrict the population to adults and the upper cutoff was used because of potential eligibility for Health Insurance for the Elderly); (5) finally, individuals who were inpatients at the time of the index date were excluded from the analysis.

\section{Outcome measures}

Past observational research has differentiated antipsychotic monotherapy from antipsychotic polypharmacy in multiple ways. ${ }^{27}$ The simplest definition of antipsychotic polypharmacy is the use of two or more antipsychotics on any given day. Unfortunately, this elegant definition also includes crosstapering and "as needed" use. A more restrictive definition is the concurrent use of two or more antipsychotics for at least 60 days. ${ }^{27}$ This restrictive definition eliminates cross-tapering and "as needed" use, but it also excludes any short-term polypharmacy.

The primary outcome variable in this study was the proportion of patients treated with monotherapy across each day of the treatment period. The second outcome variable, duration of monotherapy, was defined as the number of days of antipsychotic monotherapy use from the index date to the end of the study. The final outcome variable was defined as the time in days from the index date to the first day of a period of persistent antipsychotic polypharmacy (60+ days). The first two outcome variables use a simple definition of polypharmacy while also incorporating time and the third outcome variable captures only the more restrictive definition of polypharmacy.

\section{Baseline measures}

All analyses were adjusted for baseline differences between patients initiated on olanzapine and patients initiated on risperidone. The baseline variables were calculated from information available during the 6 months prior to the index date.

The Anatomical Therapeutic Chemical Classification System codes from the pharmacy claims were used to create indicator (yes/no) variables for baseline treatment with several relevant classes of drugs. Atypical antipsychotics were defined as olanzapine, risperidone, aripiprazole, blonanserin, clozapine, perospirone, quetiapine, and zotepine. Anticholinergic/ antiparkinsonian drugs included amantadine, biperiden, levodopa, carbidopa-levodopa, selegiline, and trihexyphenidyl.
Antidepressants included amoxapine, setiptiline, trimipramine, mirtazapine, amitriptyline, imipramine, clomipramine, trazodone, dosulepin, nortriptyline, maprotiline, mianserin, lofepramine, milnacipran, fluvoxamine, sertraline, and paroxetine. Hypnotics/sedatives included amobarbital, secobarbital, barbital, phenobarbital, pentobarbital, passiflora, estazolam, quazepam, zopiclone, triazolam, trichloroethyl, nitrazepam, nimetazepam, haloxazolam, flunitrazepam, flurazepam, brotizolam, bromvalerylurea, lormetazepam, rilmazafone, zolpidem, and chloral hydrate. The final baseline pharmacy variables were an indicator variable for prior use of risperidone and a second indicator variable for prior use of olanzapine.

Baseline comorbidities were identified using the ICD-10 diagnostic codes. Indicator variables for the following comorbid conditions were coded: depression (F32, F33), manic episodes (F30), and diabetes mellitus (E10-E14).

Any physician the patient consulted could have written the initial prescription for the index drug (olanzapine or risperidone). If the physician was identified as a psychiatrist in the administrative claims, the index drug was considered "psychiatrist prescribed."

Health care utilization variables were created to capture the costs and resources used by patients during the baseline period. Outpatient visits were measured as a count of the number of outpatient visits. Inpatient service utilization was coded as present or absent. Antipsychotic adherence was designated if a patient had filled scripts for any antipsychotic on $80 \%$ or more of the days in the baseline period (ie, medication possession ratio $\geq 0.80$ ). Finally, total health care costs were aggregated for the baseline period, based on the amounts paid by the health plans for medical services and medication prescriptions.

\section{Statistical methods}

Univariate comparisons of baseline characteristics between patients initiated on olanzapine or risperidone were conducted using chi-square tests or Fisher's exact tests for categorical variables and Student's $t$-tests or Wilcoxon rank-sum tests for continuous variables. A propensity score predicting the probability of initiating treatment with olanzapine or risperidone was calculated using logistic regression with the baseline variables. Balance between the treatment groups on each baseline characteristic after adjusting for the propensity score was verified using analysis of variance for continuous variables and logistic regression for categorical variables. Table 1 contains each of the variables used in the propensity score calculation. 
Table I Baseline characteristics for olanzapine and risperidone initiators

\begin{tabular}{|c|c|c|c|}
\hline Variable & Olanzapine $(n=332)$ & Risperidone $(n=496)$ & $P$-value \\
\hline \multicolumn{4}{|l|}{ Demographics } \\
\hline Age (years) [mean (SD)] & $36.5(9.8)$ & $37.2(10.6)$ & 0.35 \\
\hline Gender (male) [n (\%)] & $163(49.1)$ & $234(47.2)$ & 0.59 \\
\hline \multicolumn{4}{|l|}{ Prior resource use } \\
\hline Inpatient service use [n (\%)] & $27(8.1)$ & $46(9.3)$ & 0.57 \\
\hline Outpatient visits [mean (SD)] & $16.0(14.4)$ & $16.4(17.0)$ & 0.68 \\
\hline Total treatment cost (¥) [mean (SD)] & $208,894(335,316)$ & $235,500(340,121)$ & 0.77 \\
\hline Antipsychotic adherence [n (\%)] & $95(28.6)$ & I7| (34.5) & 0.08 \\
\hline \multicolumn{4}{|l|}{ Prior comorbidities } \\
\hline Diabetes [n (\%)] & $36(10.8)$ & $79(15.9)$ & 0.04 \\
\hline Depression [n (\%)] & I58 (47.6) & $218(44.0)$ & 0.30 \\
\hline Manic episode [n (\%)] & $197(59.3)$ & $254(5 \mathrm{I} .2)$ & 0.02 \\
\hline \multicolumn{4}{|l|}{ Prior medication use } \\
\hline Atypical antipsychotic use [n (\%)] & $145(43.7)$ & $237(47.8)$ & 0.25 \\
\hline Typical antipsychotic use [n (\%)] & $106(31.9)$ & $184(37.1)$ & 0.13 \\
\hline Olanzapine use $[\mathrm{n}(\%)]$ & $52(15.7)$ & $92(18.5)$ & 0.28 \\
\hline Risperidone use $[\mathrm{n}(\%)]$ & $75(22.6)$ & $117(23.6)$ & 0.74 \\
\hline Antipsychotic polypharmacy use [n (\%)] & $44(13.3)$ & $90(\mid 8.1)$ & 0.06 \\
\hline Antidepressant use $[\mathrm{n}(\%)]$ & $188(56.6)$ & $215(43.3)$ & $<0.001$ \\
\hline Hypnotics/sedative use [n (\%)] & $183(55.1)$ & $270(54.4)$ & 0.85 \\
\hline Anticholinergic use [n (\%)] & $69(20.8)$ & I $35(27.2)$ & 0.04 \\
\hline Psychiatrist prescribed [n (\%)] & I $34(40.4)$ & $186(37.5)$ & 0.41 \\
\hline
\end{tabular}

Abbreviation: SD, standard deviation.

In each of the outcome models, the propensity score was included as a covariate to adjust for background difference. The probability of monotherapy across the 12-month outcome period was estimated using a repeated-measures generalized estimating equation model with a logit link function, an autoregressive error structure, and a binomial error distribution. This model included terms for treatment (olanzapine or risperidone), time (in days), the treatment-bytime interaction, and the propensity score.

Duration of monotherapy was a positively skewed variable; therefore, a nonparametric, propensity score bin bootstrapping resampling approach was used to test difference in duration between olanzapine and risperidone. Finally, survival analyses compared the time to persistent polypharmacy between olanzapine- and risperidone-initiated patients. The time to persistent polypharmacy was compared using the Kaplan-Meier method with a log-rank test and a Cox proportional hazards model that also included the propensity score. Survival curves were constructed from unadjusted Kaplan-Meier estimates. In the survival analyses, patients were censored (no longer eligible to be considered for a "treated with persistent antipsychotic polypharmacy" event) if they discontinued the index drug for a period of 30 or more days or if they completed the full 12-month study period with monotherapy. The significance level was set at $\alpha=0.05$ for all hypothesis tests and all analyses were conducted using SAS software (v 9.1.3; SAS Institute Inc, Cary, NC).

\section{Results}

Figure 1 displays a flow diagram for the olanzapine- and risperidone-treatment cohorts following the application of each inclusion and exclusion criteria. Nearly two-thirds $(63.7 \%)$ of the individuals with schizophrenia in the initial JMDC data had at least one pharmacy claim for olanzapine or risperidone. After applying all inclusion and exclusion criteria, the final analytic sample consisted of 828 individuals -332 were treated with olanzapine and

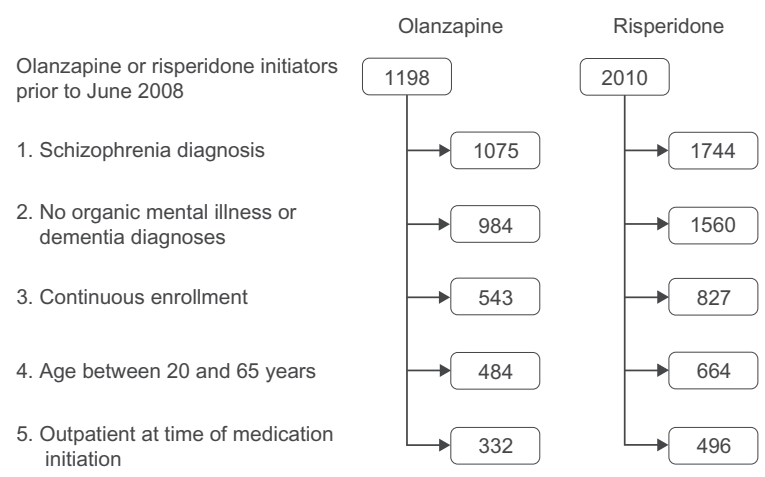

Figure I Patient selection. The diagram displays the number of olanzapine and risperidone initiators remaining after each of the inclusion and exclusion criteria were applied. The continuous enrollment criteria required each patient to have at least one claim prior to and following the study period. 
496 treated with risperidone. The average age of the patients in the final sample was 36.9 years, and $48.0 \%$ were male.

Table 1 provides the baseline demographic characteristics, prior health care resource use, prior medical and psychiatric comorbidities, and prior medication use and univariate comparison between the olanzapine and risperidone cohorts. The risperidone initiators were more likely to have prior claims with diagnoses for diabetes mellitus and prior use of anticholinergic drugs. The olanzapine initiators were more likely to have prior claims with diagnoses for manic episodes and prior antidepressant use. The $C$-statistic for the logistic regression used to calculate the propensity score from all of the characteristics in Table 1 was 0.608 , indicating that the model could accurately discriminate a randomly selected risperidone-treated individual from a randomly selected olanzapine-treated individual $60.8 \%$ of the time.

On the index date, $61.5 \%$ of the olanzapine-initiated patients and $45.6 \%$ of the risperidone-initiated patients were treated with antipsychotic monotherapy $(P<0.001)$. For the patients who were treated with antipsychotic polypharmacy, Table 2 lists the antipsychotics that were used as initial polypharmacy with olanzapine or risperidone.

Figure 2 displays the percent of olanzapine and risperidone initiated patients who were treated with antipsychotic monotherapy during the 12-month treatment period. Table 3 gives the results of the generalized estimating equation model, comparing the rates of monotherapy over time after adjusting for baseline differences. Across the 12-month treatment period, olanzapine-treated patients were more likely to be treated with monotherapy than risperidone-treated

Table 2 Initial concomitant antipsychotic treatment

\begin{tabular}{|c|c|c|c|c|}
\hline \multirow[t]{2}{*}{ Antipsychotic } & \multicolumn{2}{|c|}{ Olanzapine } & \multicolumn{2}{|c|}{ Risperidone } \\
\hline & $\mathbf{n}$ & $\% *$ & $\mathbf{n}$ & $\% *$ \\
\hline Aripiprazole & 30 & 9.0 & 55 & II.I \\
\hline Haloperidol & 23 & 6.9 & 42 & 8.5 \\
\hline Olanzapine & N/A & $\mathrm{N} / \mathrm{A}$ & 59 & 11.9 \\
\hline Quetiapine & 15 & 4.5 & 19 & 3.8 \\
\hline Levomepromazine & 10 & 3.0 & 17 & 3.4 \\
\hline Risperidone & 18 & 5.4 & $\mathrm{~N} / \mathrm{A}$ & N/A \\
\hline $\begin{array}{l}\text { Chlorpromazine/promethazine/ } \\
\text { phenobarbital }\end{array}$ & 5 & 1.5 & 12 & 2.4 \\
\hline Chlorpromazine hydrochloride & 2 & 0.6 & 8 & 1.6 \\
\hline Zotepine & 3 & 0.9 & 12 & 2.4 \\
\hline Sulpiride & 4 & 1.2 & 8 & 1.6 \\
\hline Bromperidol & 2 & 0.6 & 9 & 1.8 \\
\hline Perospirone & 5 & 1.5 & 5 & 1.0 \\
\hline Other antipsychotics & II & 3.3 & 24 & 4.8 \\
\hline
\end{tabular}

Note: *Denominators for the percentages were 332 for the olanzapine cohort and 496 for the risperidone cohort.

Abbreviation: N/A, not applicable.

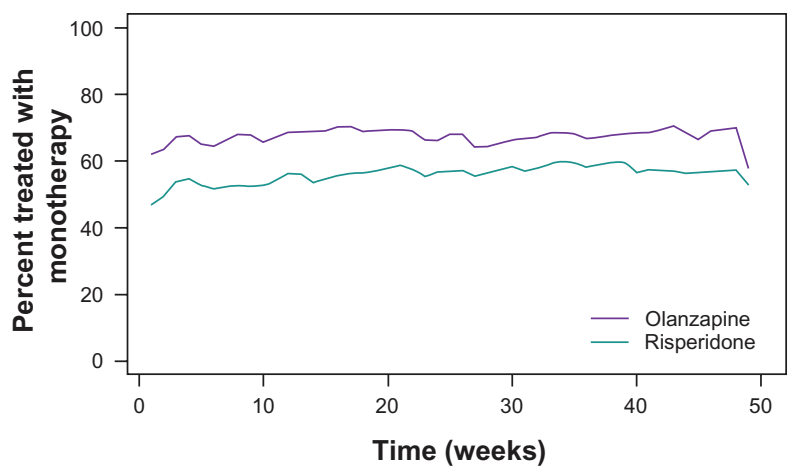

Figure 2 Percentage of patients treated with monotherapy during the 12 months following initiation of olanzapine or risperidone. The generalized estimating equation model showed that, after correcting for baseline characteristics, patients initiated on olanzapine were significantly more likely to be treated with monotherapy across the I2-month treatment period $(P=0.00 \mathrm{I})$ and the rate of monotherapy increased over time $(P<0.001)$.

patients, and the rates of monotherapy increased over time for both treatment groups.

The mean (plus or minus the standard deviation) duration of monotherapy was $116.6 \pm 130.6$ days for the olanzapinetreated patients and $92.8 \pm 123.0$ days for the risperidonetreated patients $(P=0.008)$. The propensity score-adjusted bootstrapping model showed that olanzapine-treated patients were treated with monotherapy for 22.2 (95\% confidence interval: 5.0, 39.8) days longer than risperidone-treated patients $(P=0.006)$.

Finally, a time-to-event model was used to contrast the time to persistent polypharmacy for olanzapine- and risperidone-initiated patients. Figure 3 gives the KaplanMeier estimates of the proportion of patients remaining on monotherapy across the 12-month study period. In a Cox proportional hazards model, adjusting for background differences using the propensity score, the hazard ratio for antipsychotic polypharmacy use in the olanzapine-treated patients relative to risperidone-treated patients was 0.72 (95\% confidence interval: $0.54,0.97$; chi-square $=4.71$; $P<0.03)$. The hazard ratio indicates the odds were 0.72 that an olanzapine-treated patient would utilize polypharmacy before a risperidone-treated patient.

\section{Discussion}

Consistent with prior observational research in Japan, antipsychotic polypharmacy was common in this naturalistic sample of outpatients with schizophrenia. A univariate analysis showed that the patients treated with olanzapine (61.5\%) were more likely to be initiated on antipsychotic monotherapy than those treated with risperidone $(45.6 \%, P<0.001)$. When adjusting for background characteristics and examining the 
Table 3 Results of generalized estimating equation model predicting probability of monotherapy over the 12-month study

\begin{tabular}{llllll}
\hline Effect & Estimate & $\mathbf{9 5 \%} \mathbf{~ C l}$ & OR & $\chi^{2}$ & P-value \\
\hline Antipsychotic (olanzapine) & 0.483 & $0.203,0.763$ & 1.621 & 11.55 & $<0.001$ \\
Time (days) & 0.0012 & $0.0005,0.0019$ & 1.0012 & 9.73 & 0.002 \\
Antipsychotic* time (days) & -0.0006 & $-0.0018,0.0007$ & 0.9994 & 0.75 & 0.386 \\
Propensity score & -4.960 & $-6.469,-3.450$ & 0.007 & 44.89 & $<0.001$ \\
\hline
\end{tabular}

Notes: *The antipsychotic effect indicated the odds of olanzapine being used as monotherapy was I.62I times higher than risperidone. The time effect indicates that monotherapy use increased over each day in the follow-up period. The non-significant antipsychotic-by-time interaction indicated that the difference between olanzapine and risperidone did not change over time more than was expected by chance.

Abbreviations: $\chi^{2}$, chi-square; $\mathrm{Cl}$, confidence interval; OR, odds ratio.

probability of antipsychotic monotherapy across the full 12-month study period, the patients treated with olanzapine were significantly more likely to be treated with monotherapy (odds ratio $[\mathrm{OR}]=1.62$ ) and the rate of monotherapy increased over time for both treatment groups. Additionally, the duration of antipsychotic monotherapy was significantly longer for olanzapine- than risperidone-treated patients. In Japan, where antipsychotic polypharmacy is particularly common in the treatment of schizophrenia, olanzapine treatment was associated with a significantly greater rate and a longer duration of monotherapy than risperidone treatment.

Greater use of antipsychotic monotherapy for patients using olanzapine than risperidone has previously been found in naturalistic studies outside of Japan. ${ }^{27,32}$ In a large observational study in the US, olanzapine was found to be significantly $(\mathrm{OR}=1.36)$ more likely to be used as monotherapy than risperidone and for a significantly longer duration ( 21.5 days longer; 252.1 vs 230.6 days). ${ }^{27}$ The difference in duration found in the current study (22.5 days longer) was very similar. In the Pan-European Schizophrenia Outpatient Health Outcomes study, the odds of monotherapy for olanzapine treatment relative to risperidone were 1.56, remarkably similar to the current study $(\mathrm{OR}=1.62)$, with significantly more days of monotherapy for olanzapine- than

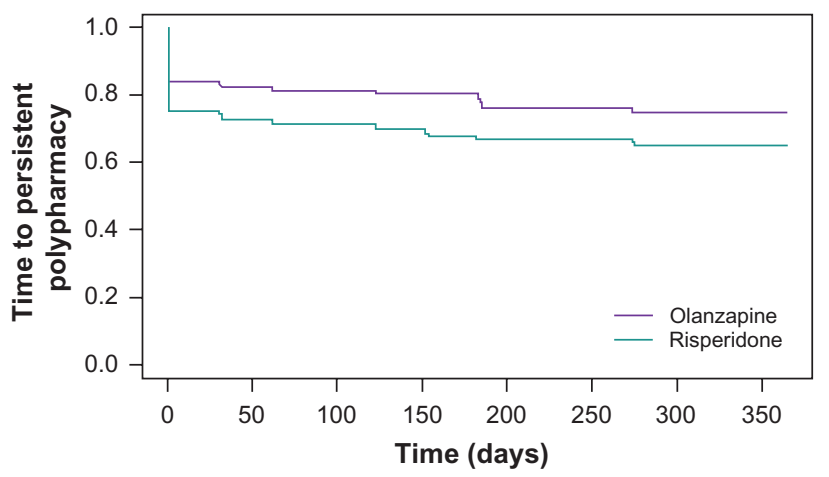

Figure 3 Time to persistent antipsychotic polypharmacy for olanzapine- and risperidone-initiated patients. The log-rank test indicated that olanzapine patients had a significantly longer time to persistent polypharmacy $(P=0.002)$ than risperidone patients. risperidone-treated patients ( 272 vs 261 days).$^{32}$ The findings in the current Japanese study replicate the findings previously reported in the US and Europe. However, the duration of monotherapy was shorter in this Japanese study (116.6 and 92.8 days for olanzapine and risperidone, respectively), which is consistent with prior research reporting more frequent use of polypharmacy in Japan. ${ }^{36}$

The finding that olanzapine was associated with greater antipsychotic monotherapy use was not unanticipated, considering that antipsychotic polypharmacy may be implemented to bolster medication efficacy. ${ }^{10,11}$ A meta-analysis of 78 studies with 13,558 participants found olanzapine to confer significantly greater efficacy than other atypicals, including risperidone, in the treatment of schizophrenia. ${ }^{37}$ The results of this meta-analysis were rather robust with regard to the effects of industry sponsorship, study quality, dosages, and trial duration. Greater effectiveness of olanzapine than risperidone in the treatment of schizophrenia offers a potential explanation of the current findings. However, the reasons physicians chose to use antipsychotic monotherapy or polypharmacy for each patient could not be specifically examined in this claims database study. Physicians may prefer to use risperidone as polypharmacy because of other properties of the medications or for patients presenting with a specific set of symptoms.

\section{Limitations}

Administrative claims data are collected for reimbursement rather than clinical purposes, thus the accuracy and level of detail are limited. Many conditions are not coded in claims and some comorbid conditions might have been underdiagnosed. The percentage of patients with claims for diabetes mellitus was higher than the $8.6 \%$ prevalence rate reported for patients with schizophrenia in Japan from past chart review research. ${ }^{38}$ Diabetes mellitus diagnoses may be coded on a claim any time a blood test (ie, HbA1c) is conducted, regardless of the status of the results, leading to the potential for overdiagnosis. 
Retrospective analyses of administrative claims data allow for the unobtrusive observation of usual clinical care at the cost of experimental control. Patients were not randomized to olanzapine or risperidone. Although propensity scores were used to adjust for multiple background variables, differences between olanzapine- and risperidone-initiated patients on variables not collected in this database could still bias the results. For instance, physician characteristics and preferences were not available to be included in the propensity score model. Finally, because the administrative data contained claims only for employees or their families, the sample was younger and might not be representative of the Japanese population as a whole.

\section{Conclusion}

Consistent with prior global research, this retrospective naturalistic study of outpatients with schizophrenia in Japan found that olanzapine is more likely than risperidone to be used as monotherapy and for a longer duration. Antipsychotic monotherapy is consistently recommended over polypharmacy because of reduced complexity, reduced risk of adverse events, reduced risk of drug interactions, and reduced total cost of drug therapy.

\section{Acknowledgments}

Eli Lilly and Company, Indianapolis, IN, provided funding for this study. Michael Stensland of Agile Outcomes Research Inc, Rochester, MN, and Susan Dennett of Strategic Health Outcomes Inc, Carmel, IN, provided technical writing support.

\section{Disclosure}

Wenyu Ye, Yuka Tanji, Jennifer Flynn, and Michihiro Takahashi are full-time employees of Eli Lilly Japan KK, Kobe, Japan. Haya Ascher-Svanum and Robert Conley are full-time employees of Eli Lilly and Company. All authors are minor stockholders in Eli Lilly and Company.

\section{References}

1. Lehman AF, Lieberman JA, Dixon LB, et al. Practice guideline for the treatment of patients with schizophrenia, 2nd ed. Am J Psychiatry. 2004;161(Suppl 2):1-56.

2. Rittmannsberger H, Meise U, Schauflinger K, Horvath E, Donat H, Hinterhuber H. Polypharmacy in psychiatric treatment: patterns of psychotropic drug use in Austrian psychiatric clinics. Eur Psychiatry. 1999;14(1):33-40.

3. Williams CL, Johnstone BM, Kesterson JG, Javor KA, Schmetzer AD. Evaluation of antipsychotic and concomitant medication use patterns in patients with schizophrenia. Med Care. 1999;37(4 Suppl Lilly): AS81-AS86.

4. Millier A, Sarlon E, Azorin JM, et al. Relapse according to antipsychotic treatment in schizophrenic patients: a propensity-adjusted analysis. $B M C$ Psychiatry. 2011;11:24.
5. Schumacher JE, Makela EH, Griffin HR. Multiple antipsychotic medication prescribing patterns. Ann Pharmacother. 2003;37(7-8): 951-955.

6. Tapp A, Wood AE, Secrest L, Erdmann J, Cubberley L, Kilzieh N. Combination antipsychotic therapy in clinical practice. Psychiatr Serv. 2003;54(1):55-59.

7. Ganguly R, Kotzan JA, Miller LS, Kennedy K, Martin BC. Prevalence, trends, and factors associated with antipsychotic polypharmacy among Medicaid-eligible schizophrenia patients, 1998-2000. JClin Psychiatry. 2004;65(10):1377-1388.

8. Ghaemi SN. "All the worse for the fishes": conceptual and historical background of polypharmacy in psychiatry. In: Ghaemi SN, editor. Polypharmacy in Psychiatry. New York (NY): Marcel Dekker; 2002: 1-34.

9. Oepen G. Polypharamcy in schizophrenia. In: Ghaemi SN, editor. Polypharmacy in Psychiatry. New York (NY): Marcel Dekker; 2002:101-132.

10. Correll CU. Antipsychotic polypharmacy, part 1: shotgun approach or targeted cotreatment? J Clin Psychiatry. 2008;69(4):674-675.

11. Barnes TR, Paton C. Antipsychotic polypharmacy in schizophrenia: benefits and risks. CNS Drugs. 2011;25(5):383-399.

12. Freudenreich O, Goff DC. Antipsychotic combination therapy in schizophrenia: a review of efficacy and risks of current combinations. Acta Psychiatr Scand. 2002;106(5):323-330.

13. Advokat C, Dixon D, Schneider J, Comaty JE Jr. Comparison of risperidone and olanzapine as used under "real-world" conditions in a state psychiatric hospital. Prog Neuropsychopharmacol Biol Psychiatry. 2004;28(3):487-495.

14. Miller AL, Craig CS. Combination antipsychotics: pros, cons, and questions. Schizophr Bull. 2002;28(1):105-109.

15. Loosbrock DL, Zhao Z, Johnstone BM, Morris LS. Antipsychotic medication use patterns and associated costs of care for individuals with schizophrenia. J Ment Health Policy Econ. 2003;6(2):67-75.

16. Jaffe AB, Levine J. Antipsychotic medication coprescribing in a large state hospital system. Pharmacoepidemiol Drug Saf. 2003;12(1): $41-48$.

17. Zhu B, Ascher-Svanum H, Faries DE, Correll CU, Kane JM. Cost of antipsychotic polypharmacy in the treatment of schizophrenia. $B M C$ Psychiatry. 2008;8:19.

18. Rupnow MF, Greenspan A, Kosik-Gonzalez C, Zhu Y, Garawabi G, Stahl SM. Use and cost of polypharmacy in schizophrenia: data from a randomized double-blind study of risperidone and quetiapine. Value Health. 2005;8:399.

19. Stahl SM. Antipsychotic polypharmacy: squandering precious resources? J Clin Psychiatry. 2002;63(2):93-94.

20. Procyshyn RM, Kennedy NB, Tse G, Thompson B. Antipsychotic polypharmacy: a survey of discharge prescriptions from a tertiary care psychiatric institution. Can J Psychiatry. 2001;46(4):334-339.

21. Clark RE, Bartels SJ, Mellman TA, Peacock WJ. Recent trends in antipsychotic combination therapy of schizophrenia and schizoaffective disorders: implications for state mental health policy. Schizophr Bull. 2002;28(1):75-84.

22. Covell NH, Jackson CT, Evans AC, Essock SM. Antipsychotic prescribing practices in Connecticut's public mental health system: rates of changing medications and prescribing styles. Schizophr Bull. 2002;28(1):17-29.

23. Jerrell JM. Cost-effectiveness of risperidone, olanzapine, and conventional antipsychotic medications. Schizophr Bull. 2002;28(4):589-605.

24. Centorrino F, Goren JL, Hennen J, Salvatore P, Kelleher JP, Baldessarini RJ. Multiple versus single antipsychotic agents for hospitalized psychiatric patients: case-control study of risks versus benefits. Am J Psychiatry. 2004;161(4):700-706.

25. Yoshi T, Kurosawa M, Sugimura K, et al. Current status of the psychopharmacological treatment of schizophrenic patients in Japan: from 2005 nationwide survey of 9 psychiatric hospitals in the psychiatric clinical pharmacy society. Jpn J Clin Psychopharmacol. 2007;10: 1721-1731. 
26. Yoshi T, Uno J, Nakagawa M, et al. Survey of the prescription for psychotherapy in Japanese inpatients with schizophrenia in 2006. Jpn J Clin Psychopharmacol. 2010;13:1535-1545.

27. Faries D, Ascher-Svanum H, Zhu B, Correll C, Kane J. Antipsychotic monotherapy and polypharmacy in the naturalistic treatment of schizophrenia with atypical antipsychotics. BMC Psychiatry. 2005;5:26.

28. Bitter I, Chou JC, Ungvari GS, et al. Prescribing for inpatients with schizophrenia: an international multi-center comparative study. Pharmacopsychiatry. 2003;36(4):143-149.

29. Correll CU, Kane JM, O’Shea D, Razi K, Malhotra AK. Antipsychotic polypharmacy in the treatment of schizophrenia. Schizophr Res. 2003;60(1 Suppl 1):S37.

30. Wang PF, Zhao Z. Comparison of olanzapine versus quetiapine in the treatment of hospitalized patients with schizophrenia. Value Health. 2003;6:355.

31. Correll CU, Frederickson AM, Kane JM, Manu P. Does antipsychotic polypharmacy increase the risk for metabolic syndrome? Schizophr Res. 2007;89(1-3):91-100.

32. Novick D, Ascher-Svanum H, Brugnoli R, Bertsch J, Hong J, Haro JM. Antipsychotic monotherapy treatment with atypicals in outpatients with schizophrenia: results from a naturalistic observational study. Schizophr Bull. 2011;37(Suppl 1):S316-S317

33. Yoshimura R, Okamoto T, Nakamura J, et al. Prescription pattern of antipsychotic drugs for schizophrenic inpatients in Japan: research on East Asia Psychotropic Prescription Pattern-Antipsychotics study. Psychiatry Clin Neurosci. 2006;60(6):778-779.
34. World Health Organization. International Statistical Classification of Diseases and Related Health Problems 10th Revision version for 2007. 2nd ed. 2007. Available from: http://apps.who.int/classifications/apps/ icd/icd10online2007/. Accessed January 20, 2011.

35. World Health Organization Collaborating Centre for Drug Statistics Methodology. World Health Organization Anatomical Therapeutic Chemical (ATC) classification index including defined daily doses (DDDs). Oslo, Norway: World Health Organization Collaborating Centre for Drug Statistics Methodology. Available from: http://www. whocc.no/atc_ddd_index/. Accessed January 20, 2011.

36. Shinfuku N, Tan $\mathrm{CH}$. Pharmacotherapy for schizophrenic inpatients in East Asia: changes and challenges. Int Rev Psychiatry. 2008;20(5): 460-468.

37. Leucht S, Komossa K, Rummel-Kluge C, et al. A meta-analysis of head-to-head comparisons of second-generation antipsychotics in the treatment of schizophrenia. Am J Psychiatry. 2009;166(2):152-163.

38. Okumura Y, Ito H, Kobayashi M, Mayahara K, Matsumoto Y, Hirakawa J. Prevalence of diabetes and antipsychotic prescription patterns in patients with schizophrenia: a nationwide retrospective cohort study. Schizophr Res. 2010;119(1-3):145-152.
Neuropsychiatric Disease and Treatment

\section{Publish your work in this journal}

Neuropsychiatric Disease and Treatment is an international, peerreviewed journal of clinical therapeutics and pharmacology focusing on concise rapid reporting of clinical or pre-clinical studies on a range of neuropsychiatric and neurological disorders. This journal is indexed on PubMed Central, the 'PsycINFO' database and CAS.

\section{Dovepress}

The manuscript management system is completely online and includes a very quick and fair peer-review system, which is all easy to use. Visit $\mathrm{http}: / / \mathrm{www}$.dovepress.com/testimonials.php to read real quotes from published authors. 